ISSN: 2600-5859

\title{
La gestión financiera para el desarrollo competitivo de las cooperativas de ahorro y credito.
}

\section{Financial management for the competitive development of credit unions.}

Sandra Patricia Jácome Tamayo. 1, María Isabel Gavilánez Vega. ² María del Carmen Ibarra Chango. ${ }^{3}$

\section{Abstract. \\ DOI: https://doi.org/10.33262/concienciadigital.v3i1.1.1154}

Currently, the Ecuadorian financial cooperative sector is very competitive, where the offer of financial and non-financial products and services vary in their specialization and innovation, becoming drivers of financial, economic and competitiveness management, differentiating them in quality, quantity and cost. that maintain a balance with each other, therefore in order to determine how financial management affects the competitive development of credit unions in the present investigation correlates the administration, finance, management and control of capital flows monetary and financial; for which theoretical and empirical methods were used through the application of financial tools and strategies such as horizontal, vertical analysis, ratios and evaluation methodology PEARLS, whose basis of analysis are the balance sheets and financial statements of different cycles, it is highlighted that the Structured and systematized analysis delineates the pattern of

\footnotetext{
${ }^{1}$ Escuela Superior Politécnica de Chimborazo, Facultad de Administración de Empresas, Riobamba, Ecuador, sandra.jacome@espoch.edu.ec

${ }^{2}$ Escuela Superior Politécnica de Chimborazo, Facultad de Administración de Empresas, Riobamba, Ecuador, maria.gavilanez@espoch.edu.ec

${ }^{3}$ Escuela Superior Politécnica de Chimborazo, Facultad de Administración de Empresas, Riobamba, Ecuador, mariadelcarmen.ibarra@espoch.edu.ec
} 
integrated growth with a long-term vision, in order to promote planned results which serve as the basis for future analysis and starting point for research that develops continuous improvements.

Keywords: Management, financial indexes, finance, financial analysis.

\section{Resumen.}

Actualmente el sector cooperativo financiero ecuatoriano es muy competitivo, donde la oferta de productos y servicios financieros y no financieros varían en su especialización e innovación, constituyéndose en factores impulsadores de la gestión financiera, económica y de la competitividad, diferenciándolos en calidad, cantidad y costo que mantienen un equilibrio entre sí, por tanto con el objeto de determinar cómo la gestión financiera incide en el desarrollo competitivo de las Cooperativas de ahorro y crédito en la presente investigación se correlaciona la administración, las finanzas, el manejo y control de flujos de capitales monetarios y financieros; para lo cual se usó métodos teóricos y empíricos mediante la aplicación de herramientas y estrategias financieras tales como análisis horizontal, vertical, ratios y metodología de evaluación PERLAS, cuya base de análisis son los balances y estados financieros referenciales de diferentes ciclos, se destaca que el análisis estructurado y sistematizado delinea el patrón de crecimiento integrado con una visión a largo plazo, en post de promover resultados planificados lo cuales sirvan como base para futuros análisis y punto de partida de investigaciones que desarrollen mejoras continuas.

Palabras claves: Gestión, índices financieros, finanzas, análisis financiero.

\section{Introducción.}

Para promover una correcta administración y control de los flujos de capitales monetarios y financieros, en el Ecuador el Sistema Financiero y Monetario actualmente está regulado por 
el Código Orgánico Monetario Financiero, normativa que busca generar un nuevo modelo de gestión financiera.

Actualmente en el Ecuador, el organismo encargado del control de las cooperativas de ahorro y crédito (COAC), es el la Superintendencia de Economía Popular y Solidaria (SEPS), institución que ha evolucionado según las exigencias competitivas del mercado, destacándose que en la actualidad existe más de 7.4 millones de personas que fungen como socios de alguna COAC; destacándose, que según criterio de las personas sienten que en este tipo de organizaciones financieras es más fácil realizar créditos en comparación con los bancos.

Es decir, desde el año 2012 hasta la actualidad, el número de socios en la COAC se ha incrementado en un 51\%; lo cual ha dado lugar a que el patrimonio de estas instituciones se incremente; crecimiento galopante de estas organizaciones que, si se excluye a los cinco bancos privados más grandes del país, los activos, la cartera de créditos y los depósitos del sistema financiero popular y solidario son mayores a los del sistema bancario, tal como lo destaca Diario el Universo en su edición del 19 de mayo del 2019.

Mercado que se ha vuelto altamente competitivo por lo cual las COAC han implementado nuevos servicios para que se adapten a las diversas exigencias, características o particularidades de los socios y clientes, presentando una gama diversa de productos financieros altamente competitivos, diversificando el mercado, pero al mismo tiempo generando una alta vulnerabilidad y la exposición a los diferentes riesgos.

Por tanto, en consideración que la COAC tiene como objetivo realizar actividades de intermediación financiera y de responsabilidad social con sus clientes, socios y con terceros, la Junta de Política y Regulación Monetaria y financiera, determino los segmentos del sector financiero tal como muestra a continuación según su número de activos. 
Tabla No1.

Segmentación de las Cooperativas de ahorro y crédito

\begin{tabular}{|c|l|}
\hline \multirow{2}{*}{ Segmento } & \multicolumn{1}{|c|}{ Activos (\$) } \\
\hline 1 & Mayor a $80^{\prime} 000.000$ \\
\hline 2 & Mayor a 20'000.000 hasta $80^{\prime} 000.000$ \\
\hline 3 & Mayor a 5'000.000 hasta 20'000.000 \\
\hline 4 & Mayor a 1'000.000,00 hasta 5'000.000 \\
\hline \multirow{2}{*}{5} & Hasta 1'000.000 \\
\cline { 2 - 2 } & Cajas de ahorro, bancos comunales y cajas comunales \\
\hline
\end{tabular}

Fuente: SEPS (2015)

Al determinar que "el primer inventario realizado por la SEPS (2012) determino que existía 946 COAC, actualmente existe 598 es decir han quebrado 348 COAC $36.78 \%$ menos de las cuales 27 pertenecen al primer segmento, 38 al segundo segmento, 86 al tercer segmento, 180 al cuarto segmento y 267 al quinto segmento; en este sentido el segmento tres, cuatro y cinco agrupan al 96\% del total de COAC a nivel nacional, sin embargo el segmento uno y dos concentran el $88,66 \%$ del total de activos y el $84,51 \%$ del total de socios del sector de COAC", tal como lo sostiene Fonseca (2019), en la evaluación económica y financiera de las Cooperativas de Ahorro y Crédito del Ecuador. Caso Segmento 2 del cantón Ambato provincia de Tungurahua.

En este mismo ámbito, a partir del 2013 la SEPS, implementa sistemas para la regulación, supervisión, monitoreo y control, coadyuvado a determinar la débil gestión institucional producto de una gestión financiera inadecuada ya que sus fuentes de financiamiento son limitadas, al igual que su planificación operativa y financiera.

Sin embargo los autores coinciden con Martínez (2016), "al determinar que el insuficiente sistema de control interno de las COAC ha generado: vulnerabilidad en la toma de decisiones de la administración; exponiendo a las entidades a excesivos riesgos financieros, lo cuales disminuyen las oportunidades y ventajas del desarrollo competitivo, 
dando lugar a la quiebra financiera provocada en el corto plazo por no existir liquidez, y en el mediano y largo plazo por la falta de rentabilidad y solvencia".

En este mismo ámbito, el entorno financiero ecuatoriano los últimos años ha presentado una serie de problemáticas resultando engañados y estafados los clientes que adquieren los productos financieros con estas entidades tanto en las tasas, como en cuota o monto a prestar Por tanto, es necesario la implementación de un modelo el cual sea el punto de partida para la implementación de la gestión por procesos en el ámbito financiero, radicando su importancia, en ser el diagrama que permita forjar indicadores y los lineamientos a seguir para la toma de decisiones. Por tanto, es indispensable la aplicación de un modelo de gestión financiera para el desarrollo competitivo de las COAC, coadyuvando a definir los límites de control, fortaleciendo al mismo tiempo la comunicación y colaboración, entre ofertantes y demandantes de servicios financieros para generar estrategias que mejoren los resultados financieros y económicos.

En este ámbito, fundamentaos en Barry (2004), Barragán (2014), es necesario que los procesos de análisis partan de la evaluación de factores internos y externos, los mismos que determinen la ubicación de cada actor en el sector financiero, y delinee la estrategia de forma oportuna y adecuada proyectándose en el futuro un crecimiento económico sostenido tendencial, para permanecer en el tercer sector económico.

Partiendo de la premisa que la planeación financiera permite generar sustentabilidad en el ámbito financiero a partir de la emisión de recomendaciones que surgen como producto de los resultados obtenido en los estudios básicos como análisis vertical, horizontal, índices de rentabilidad, solvencia y liquides, el presente estudio tiende a ir más allá para lo cual considera el análisis PERLAS.

En este ámbito según el Consejo mundial de cooperativas de ahorro y crédito (CMCOAC) el objetivo del análisis PERLAS (2009), está diseñado como una herramienta de administración que va más allá de la mera identificación de problemas, para lo cual se genera ratios 
financieras los mismos que funcionan de forma coordinada y consolidada emitiendo resultados en conjunto para la toma de decisiones.

Desde 1990, el CMCOAC emplea una serie de ratios financieros, donde cada una de las letras del termino PERLAS hace referencia a un área clave de evaluación de operaciones, tales como

Protección, Estructura financiera eficaz, Calidad de Activos, Tasas de Rendimiento y costos, Liquidez y Señales de crecimiento. ahorro y crédito, tal como se detalla a continuación.

$\mathrm{P}=$ Protección

"La protección adecuada de activos es un principio básico del nuevo modelo de cooperativas de ahorro y crédito. La protección se mide al 1) comparar la suficiencia de las provisiones para préstamos incobrables con el monto de préstamos morosos y 2) comparar las provisiones para pérdidas de inversiones con el monto total de inversiones no reguladas. La protección contra préstamos incobrables se considera adecuada si la cooperativa de ahorro y crédito tiene suficientes provisiones como para cubrir el 100\% de todos los préstamos con morosidad mayor a 12 meses, y el $35 \%$ de todos los préstamos con morosidad de 1-12 meses".

"Una protección inadecuada contra préstamos incobrables produce dos resultados indeseables: valores inflados de activos y ganancias ficticias. La mayoría de cooperativas de ahorro y crédito no desea reconocer los préstamos incobrables, y mucho menos, castigarlos contra ganancias. Esta falta de voluntad lleva a un abuso generalizado de los principios de seguridad y solidez. Se exagera el ingreso neto reportado, los valores de activos están inflados, las provisiones para préstamos incobrables son inadecuadas y los ahorros de asociados no cuentan con una protección adecuada"

Técnica que se aplica porque algunas COAC poco interés le ponen a la insuficiencia de sus provisiones para préstamos incobrables o cartera vencida porque consideran a sus reservas 
ISSN: 2600-5859

de capital como la fuente principal de protección con los préstamos incobrables lo cual es un error.

$\mathrm{E}=$ Estructura financiera eficaz

"La estructura financiera de la cooperativa de ahorro y crédito es el factor más importante en la determinación del potencial de crecimiento, la capacidad de ganancias y la fuerza financiera general; en este ámbito el sistema de PERLAS mide activos, pasivos y capital, y recomienda una estructura correcta para las COAC”,

"Activos

* el $95 \%$ de activos productivos consiste en préstamos (70-80\%) e inversiones líquidas (10-20\%)

* el 5\% de activos improductivos consiste principalmente en activos fijos (terreno, edificios, equipos, etc.)

Pasivos

el 70-80\% de depósitos de ahorro de asociados

Capital

* 10-20\% capital de aportaciones de asociados

* $10 \%$ capital institucional (reservas no distribuidas)"

$\mathrm{A}=$ Calidad de Activos

Un activo improductivo es aquel que no genera ingresos, igualmente un exceso de activos improductivos afecta las ganancias de la COAC. Por tanto, las ratios en el sistema PERLAS que se usan para identificar el impacto de los activos improductivos son:

a. Ratio de morosidad. - "Es la medida más importante que muestra la debilidad institucional. Si la morosidad está alta, normalmente afecta todas las otras áreas claves de las operaciones de COAC, la meta ideal es mantener la tasa de morosidad por debajo del 5\% del total de préstamos pendientes."

b. Porcentaje de activos improductivos. - "Mientras más alto sea este indicador, más difícil será generar ganancias suficientes. La meta también limita activos improductivos a un máximo del 5\% del total de activos de la cooperativa de ahorro y crédito 
ISSN: 2600-5859

c. Financiamiento de activos improductivos. - "Mientras la reducción del porcentaje de activos improductivos es importante, el financiamiento de estos activos es de igual importancia. Tradicionalmente, las COAC utilizan capital de aportaciones de asociados para financiar las compras de activos fijos"

$\mathrm{R}=$ Tasas de rendimiento y costos

"Se calcula el rendimiento con base en el promedio de activos, es decir, se calcula el rendimiento con base en las inversiones reales pendientes. Esta metodología ayuda a la gerencia a determinar cuáles inversiones sean las más rentables. También permite una clasificación de COAC de acuerdo con los mejores y peores rendimientos. Al comparar la estructura financiera con el rendimiento, es posible determinar qué tan eficazmente puede la COAC colocar sus recursos productivos en inversiones que producen el mayor rendimiento."

$\mathrm{L}=$ Liquidez

"El manejo eficaz de liquidez es una habilidad que tiene importancia a medida que la COAC cambia su estructura financiera de las aportaciones de asociados a los depósitos de ahorro, que son más volátiles. En muchos movimientos que siguen el modelo tradicional, las aportaciones de asociados son muy ilíquidas y la mayoría de préstamos externos tienen un largo período de reembolso. En este ámbito la liquidez tradicionalmente se analiza en términos del efectivo disponible para prestar, una variable controlada exclusivamente por la cooperativa de ahorro y crédito. Con la introducción de depósitos de ahorro que pueden ser retirados, el concepto de la liquidez cambia radicalmente. La liquidez ahora se refiere al efectivo necesario para retiros, una variable que la cooperativa de ahorro y crédito ya no puede controlar."

$\mathrm{S}=$ Señales de crecimiento

"La única manera exitosa de mantener el valor de activos es a través de un fuerte y acelerado crecimiento de activos, acompañado por la rentabilidad sostenida. El crecimiento por sí solo es insuficiente. La ventaja del sistema PERLAS es que vincula el crecimiento con la rentabilidad y con las otras áreas claves, al evaluar el crecimiento del sistema entero." 
Por tanto, el crecimiento se mide a través de cinco áreas claves, tales como los activos, prestamos, ahorros, aportaciones, y capital institucional.

Se destaca que en esta investigación se considera el sistema PERLAS por su integridad de análisis a diferencia del sistema de clasificación estadounidense CAMEL el cual no evalúa la estructura financiera del balance general y la tasa de crecimiento, ya que el mismo fue diseñado como una herramienta supervisora como una administrativa. Godoy (2010), Salgueiro (2011)

\section{Metodologia}

A partir de James (2002), Martino (2004), Amaru (2009), Gómez (2010), en el contexto de la investigación, se considera como parámetro de análisis a la COAC Kullki Wasi Ltda., para lo cual se utilizó métodos teóricos y empíricos que permita poner en ejecución el análisis PERLAS como marco de referencia para la administración financiera de la entidad, tal como se muestra en la siguiente gráfica

Gráfico No1.

Metodología para la gestión financiera

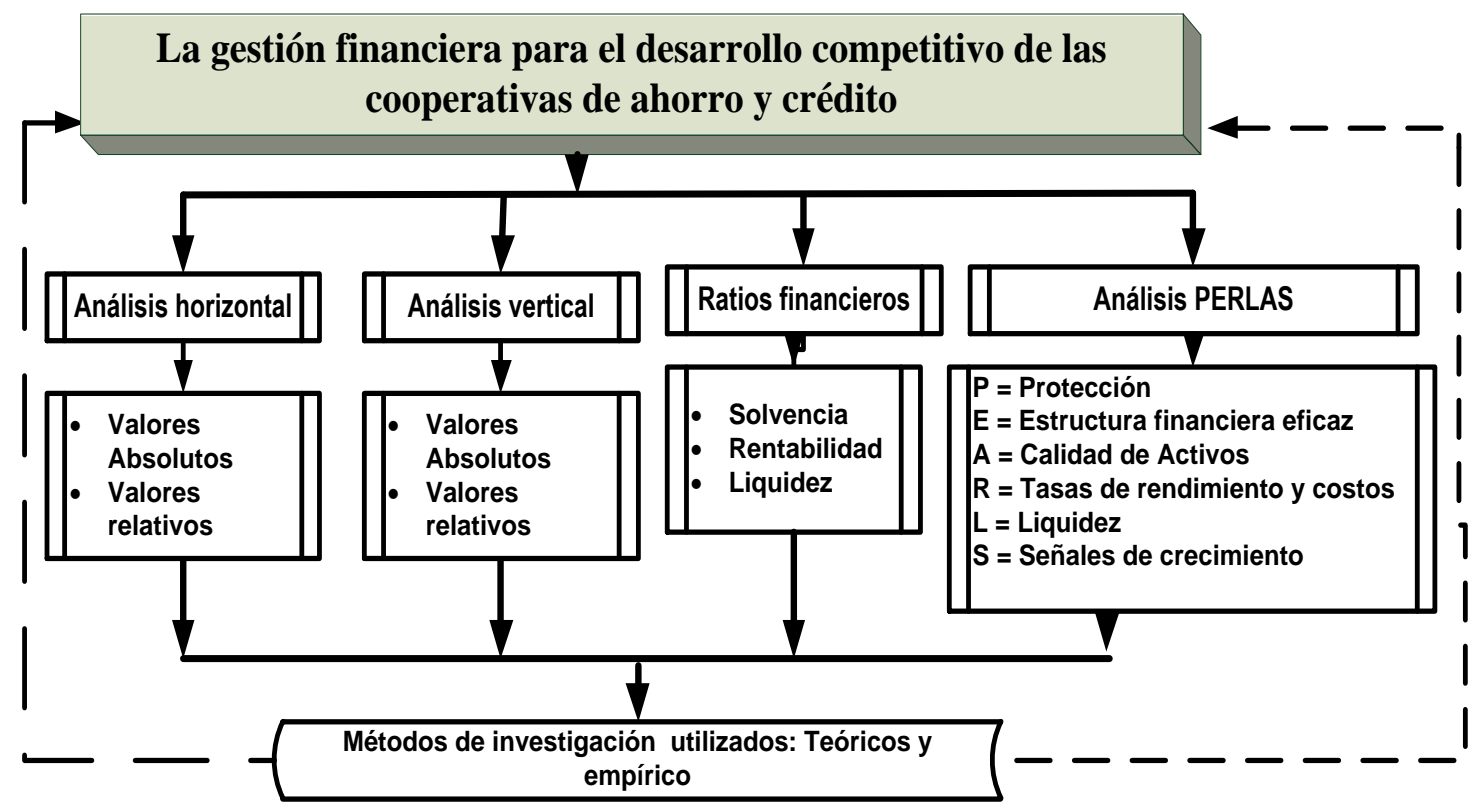

Fuente: elaborado por Jácome, S., Gavilánez, M., \& Ibarra, M. (2020) 
El modelo metodológico de investigación aplicado a la COAC "Kullki Wasi Ltda., coadyuvara despejar varias interrogantes referentes al desempeño eficiente de la gestión financiera, para lo cual se toma como referencia información financiera de los dos últimos años.

Lo cual conlleve a que pase del ámbito de la competencia a la competencia en el segmento dos donde se encuentra en la actualidad, cuyos análisis y proyecciones se enfoque en buscar llegar al segmento uno.

\section{Resultados}

En función del sistema de cuentas estandarizados en la COAC planteado por la Superintendencia de economía popular y solidaria se esquematiza el analices PERLAS acorde al alcance de las ratios, obteniéndose los siguientes resultados.

- Análisis de protección

Objetivo de medición analizar la suficiencia de provisiones para préstamos incobrables, tal como se muestra a continuación en la tabla No2.

P1.- Se determina que es mayor para préstamos incobrables en comparación con las provisiones requeridas para cubrir todos los préstamos con morosidad mayor a 12 meses. Ya que los resultados obtenidos el año 2019 superan el 100\%

P2.- Es insuficiente las provisiones para préstamos incobrables ya una vez descontado las provisiones usadas para cubrir préstamos con morosidad mayor a doce meses, los índices obtenidos de los años 2018 y 2019 son inferiores al 35\%.

Tabla No2.

Análisis de protección

\begin{tabular}{|c|c|c|c|c|}
\hline & PERLAS & 2018 & 2019 & META \\
\hline \multirow{2}{*}{ P= PROTECCIÓN } & $\begin{array}{l}\text { P1. PROVISION PARA PRÉSTAMOS INCOBRABLES/ } \\
\text { PROVISIÓN REQUERIDA } \\
\text { MOROSIDAD > } 12 \text { MESES }\end{array}$ & 0,85 & 1,96 & $100 \%$ \\
\hline & \begin{tabular}{|lcrrr} 
P2. & PROVISION & NETA & PARA & PRÉSTAMOS \\
INCOBRABLES/ & PROVISIÓN & REQUERIDA & PARA \\
PRÉSTAMOS MOROSOS $<$ A 12 & MESES & & \\
\end{tabular} & $-0,04$ & 0,15 & $35 \%$ \\
\hline
\end{tabular}

Fuente: elaborado por Jácome, S., Gavilánez, M., \& Ibarra, M. (2020)

- Estructura financiera 
Análisis generado con el objetivo de evaluar la composición de las cuentas del balance general para determinar la seguridad, solidez y rentabilidad de la COAC objeto de análisis E1.-. Al evaluar el porcentaje del activo total en relación a la cartera de préstamos se determina que se encuentra al límite de los parámetros meta fijados $70 \%$

E2.-. El activo total invertido en inversiones a corto plazo es muy eficiente los parámetros establecidos son inferiores al $4 \%$

E5.-. En promedio los años de análisis el porcentaje del activo total financiado con depósitos de ahorro es del $14 \%$ inferior a la meta sugerida entre el $70 \%-80 \%$

E6.-. Al analizar el porcentaje del activo total financiado con el crédito externo es muy eficiente ya que solo el $4 \%$ de los activos han sido financiados vía endeudamiento.

E7.-. Muestra sostenibilidad la COAC en consideración que solo el 5\% de los activos es financiado con las aportaciones de asociados.

E8.-. Se muestra leve deficiencia ya que el $11 \%$ del activo total es financiado con capital institucional.

E9.-. El capital institucional es del 27\%, después de ajustar las provisiones para activos en riesgo para satisfacer las normas de $\mathrm{P} 1 \& \mathrm{P} 2$, y cubrir cualquier otra pérdida potencial, lo cual significa que existe niveles deficientes $17 \%$ más a la norma establecida.

Tal cual como se muestra en la tabla que se muestra a continuación.

Tabla No3.

Análisis de la Estructura financiera

\begin{tabular}{|c|c|c|c|c|}
\hline & PERLAS & 2018 & 2019 & META \\
\hline \multirow{7}{*}{$\begin{array}{c}\mathrm{E}= \\
\text { EXTRUCTURA } \\
\text { FINANCIERA }\end{array}$} & E1. PRÉSTAMOS NETOS/ TOTAL ACTIVO & $72 \%$ & $70 \%$ & $70-80 \%$ \\
\hline & E2. INVERSIONES LÍQUIDAS/ TOTAL ACTIVO & 0,04 & 0,04 & Máx 20\% \\
\hline & E5. DEPÓSITOS DE AHORRO/ TOTAL ACTIVO & $15 \%$ & $13 \%$ & $70-80 \%$ \\
\hline & E6. CRÉDITO EXTERNO/ TOTAL ACTIVO & $4 \%$ & $4 \%$ & Máx el 5\% \\
\hline & E7. APORTACIONES DE ASOCIADOS/ TOTAL ACTIVO & $5 \%$ & $5 \%$ & Máx el 20\% \\
\hline & E8. CAPITAL INSTITUCIONAL TOTAL ACTIVO & $11 \%$ & $11 \%$ & Mín el 10\% \\
\hline & E9. CAPITAL INSTITUCIONAL NETO/ TOTAL ACTIVO & $27 \%$ & $27 \%$ & $\geq 10 \%$ \\
\hline
\end{tabular}

Fuente: elaborado por Jácome, S., Gavilánez, M., \& Ibarra, M. (2020)

- Tasas de rendimiento y costos

Con el objetivo de medir el costo para cada una de las cuentas de pasivos y capital más importantes, tal como se muestra en Tabla No 4. 
R1.- La evaluación de E9 es deficiente por tanto el rendimiento de la cartera de préstamos es deficiente ya que el porcentaje existente supera a la meta fijada.

R2.- El rendimiento de los depósitos en bancos, etc., en relación a las tasas más altas del mercado actualmente corren riesgo, dado la relación entre inversiones totales y las inversiones liquidas.

R5.- El rendimiento (costo) de los depósitos de ahorro es superior a la tasa de inflación actual existente en el Ecuador la cual radica por debajo del 1.4\%

R6.- El rendimiento (costo) de todo el crédito externo en promedio es del $6 \%$ superando las normas establecidas.

Tabla No 4.

Análisis de las Tasas de rendimiento y costos

\begin{tabular}{|c|c|c|c|c|}
\hline & PERLAS & 2018 & 2019 & META \\
\hline \multirow{10}{*}{$\begin{array}{l}\mathrm{R}= \\
\text { RENDIMIENTO Y } \\
\text { COSTOS }\end{array}$} & $\begin{array}{l}\text { R1. INGRESO NETO DE PRÉSTAMOS/ PROMEDIO DE } \\
\text { CARTERA DE PRÉSTAMOS NETA }\end{array}$ & $20 \%$ & $21 \%$ & $\geq 16 \%$ \\
\hline & $\begin{array}{l}\text { 2. INGRESO POR INVERSIONES LÍQUIDAS/ PROMEDIO } \\
\text { DE INVERSIONES LÍQUIDAS } \\
\end{array}$ & 0,10 & 0,13 & $4,53 \%$ \\
\hline & $\begin{array}{l}\text { R5. COSTO FINANCIERO: INTERÉSES SOBRE DEPÓSITOS } \\
\text { DE AHORRO/ PROMEDIO DE DEPÓSITOS DE AHORRO }\end{array}$ & $6 \%$ & $6 \%$ & $1,41 \%$ \\
\hline & \begin{tabular}{|llllll} 
R5.1 COSTO & FINANCIERO: & INTERÉSES & SOBRE \\
DEPÓSITOS & A & PLAZO/ & PROMEDIO & DE & DEPÓSITOS A \\
PLAZO & & & \\
\end{tabular} & $12 \%$ & $20 \%$ & $4,53 \%$ \\
\hline & $\begin{array}{l}\text { R6. COSTO FINANCIERO: INTERÉSES SOBRE EL CRÉDITO } \\
\text { EXTERNO/ PROMEDIO DE CRÉDITO EXTERNO }\end{array}$ & $8 \%$ & $5 \%$ & $5,51 \%$ \\
\hline & R8. MARGEN BRUTO/ PROMEDIO DE ACTIVO TOTAL & $15 \%$ & $15 \%$ & $\mathrm{ING}=\mathrm{GAST}$ \\
\hline & \begin{tabular}{|l} 
R9. GASTOS OPERATIVOS/ PROMEDIO DE ACTIVO \\
TOTAL
\end{tabular} & $8 \%$ & $7 \%$ & $\leq 5 \%$ \\
\hline & $\begin{array}{l}\text { R10. PROVISIONES PARA PRÉSTAMOS INCOBRABLES/ } \\
\text { PROMEDIO DE ACTIVO TOTAL }\end{array}$ & $1 \%$ & $2 \%$ & $100 \%$ y $35 \%$ \\
\hline & $\begin{array}{|lccc|}\text { R11. INGRESOS O GASTOS } & \text { EXTRAORDINARIOS/ } \\
\text { PROMEDIO DE ACTIVO TOTAL } & \\
\end{array}$ & $0,0001 \%$ & $0,016 \%$ & Minimizar \\
\hline & $\begin{array}{l}\text { R12. INGRESOS NETO/ PROMEDIO DE ACTIVO TOTAL } \\
\text { (ROA) }\end{array}$ & $0,51 \%$ & $0,06 \%$ & $>1 \%$ \\
\hline
\end{tabular}

Fuente: elaborado por Jácome, S., Gavilánez, M., \& Ibarra, M. (2020)

R8.- Existe los suficientes ingresos para cubrir todos los gastos operativos y provisiones para préstamos incobrables con lo cual se asegura el capital institucional.

R9.- Los costos vinculados con la administración de todos los activos de la COAC. Muestran ineficiencia operativa, porque su porcentaje es mayor al establecido. 
R10.- Se determina inconsistencias ya que no existe sostenibilidad para cubrir el 100\% de préstamos morosos $>12$ meses y el 35\% de préstamos morosos entre 1-12 meses.

R11.- La tendencia es contraria a la esperada porque tiene tendencia creciente ya que los resultados indican que el monto neto de ingresos es inferior a los gastos extraordinarios, sin embargo, este parámetro es insignificante porque la COAC producto de este análisis se dedica a la intermediación financiera.

R12.- Los dos periodos muestran la falta de capacidad de aumentar el capital institucional a los niveles óptimos, considerando que existe 5 puntos menos del parámetro sugerido como óptimo.

Todos los análisis responden a los resultados de la gráfica que se muestra a continuación.

\section{- Liquidez}

Los indicadores de liquidez permiten identificar que la COAC administra eficazmente su efectivo para satisfacer la demanda de retiros y reservas de liquidez del cliente.

L1.- Se determina un exceso de liquidez después de pagar todas las obligaciones inmediatas $<30$ días, ya que el porcentaje promedio de la relación activos pasivos de los dos años de análisis supera los márgenes establecidos.

L2.- Las reservas de liquidez obligatorias tipo encaje exigidas por la superintendencia son mínimos causando dificultades en el corto plazo.

L3.- La razón del activo total invertido en cuentas líquidas improductivas, es negativa a la tendencia existente en promedio es superior a la meta establecida.

Tabla No 5.

Análisis de la Liquidez

\begin{tabular}{|c|c|c|c|c|}
\hline & PERLAS & 2018 & 2019 & META \\
\hline \multirow{3}{*}{$\mathrm{L}=\mathrm{LIQUIDEZ}$} & $\begin{array}{l}\text { L1. (INVERSIONES LÍQUIDAS + ACTIVOS LÍQUIDOS - } \\
\text { CUENTAS POR PAGAR A CORTO PLAZO) / DEPÓSITOS } \\
\text { DE AHORRO }\end{array}$ & $20 \%$ & $43 \%$ & $15-20 \%$ \\
\hline & L2. RESERVAS DE LIQUIDEZ / DEPÓSITOS DE AHORRO & 1,30 & 1,27 & $10 \%$ \\
\hline & $\begin{array}{l}\text { L3. ACTIVOS LÍQUIDOS IMPRODUCTIVOS / TOTAL } \\
\text { ACTIVOS }\end{array}$ & $15 \%$ & $13 \%$ & $<1 \%$ \\
\hline
\end{tabular}

Fuente: elaborado por Jácome, S., Gavilánez, M., \& Ibarra, M. (2020)

- Señales de crecimiento 
Con el objetivo de medir las perspectivas de crecimiento de las cuentas que conforman los estados financieros se genera la correlación de ratios financieros. Se destaca que en una economía dolarizada no existe una diferencia amplia entre el PIBN y el PIBR, en consideración que en una economía dolarizada la tasa de inflación es baja.

S1.- El crecimiento de la cartera en relación a los prestamos es deficiente, porque se demuestra que R11 es inferior al establecer una evaluación cruzada de la información

S2.- Se considera indeterminada la evaluación en consideración que al analizar la estructura financiera no se considera el parámetro E3 en función del plan de cuentas existentes.

S5.- EL crecimiento del año hasta la fecha de depósitos de ahorro es deficiente ya que se muestra inferior al parámetro de sostenibilidad.

S6.- La estructura financiera muestra sostenibilidad para que exista una relación positiva la relación de créditos externos.

S7.- La estructura financiera muestra sostenibilidad para que exista una relación positiva la relación aportaciones.

S8.- La estructura financiera muestra sostenibilidad para que exista una relación positiva la relación al capital institucional

S11.- La estructura financiera muestra sostenibilidad para que exista una relación positiva la relación activo total.

Tabla No 6.

Análisis Señales de crecimiento

\begin{tabular}{|l|l|r|r|c|}
\hline & \multicolumn{1}{|c|}{ PERLAS } & $\mathbf{2 0 1 8}$ & \multicolumn{1}{c|}{$\mathbf{2 0 1 9}$} & \multicolumn{1}{c|}{ META } \\
\hline \multirow{5}{*}{$\begin{array}{l}\text { S= SENALES DE } \\
\text { CRECIMIENTO }\end{array}$} & S1. CRECIMIENTO DE PRÉSTAMOS & $18 \%$ & $31 \%$ & ${ }^{\wedge} \mathrm{E} 1=70-80 \%$ \\
\cline { 2 - 5 } & S3. CRECIMIENTO DE INVERSIONES FINANCIERAS & $62 \%$ & $29 \%$ & ${ }^{\wedge} \mathrm{E} 3 \leq 2 \%$ \\
\cline { 2 - 5 } & S5. CRECIMIENTO DE DEPÓSITOS DE AHORRO & $15 \%$ & $12 \%$ & ${ }^{\wedge} \mathrm{E} 5=70-80 \%$ \\
\cline { 2 - 5 } & S6. CRECIMIENTO DE CRÉDITO EXTERNO & $-9 \%$ & $51 \%$ & ${ }^{\wedge} \mathrm{E} 6=0-5 \%$ \\
\cline { 2 - 6 } & S7. CRECIMIENTO DE APORTACIONES DE SOCIOS & $118 \%$ & $25 \%$ & ${ }^{\wedge} \mathrm{E} 7 \leq 20 \%$ \\
\cline { 2 - 6 } & S8. CRECIMIENTO DE CAPITA INSTITUCIONAL & $58 \%$ & $25 \%$ & ${ }^{\wedge} \mathrm{E} 8 \geq 10 \%$ \\
\cline { 2 - 6 } & S11. CRECIMIENTO DEL ACTIVO TOTAL & $24 \%$ & & $>$ Inflación $+10 \%$ \\
\hline
\end{tabular}

Fuente: elaborado por Jácome, S., Gavilánez, M., \& Ibarra, M. (2020)

\section{Conclusiones:}

- El modelo de gestión financiera tiene influencia directa en el desarrollo competitivo de la COAC “Kullki Wasi Ltda.”, pues la débil gestión institucional, hace que no se 
utilicen herramientas financieras las cuales promuevan desarrollo sostenible para la institución.

- Se determina una baja cobertura de provisión de la cartera de crédito, de lo cual se idéntico que el 13\% de los activos totales has sido financiados por los depósitos de ahorros, lo cual significa que la COAC no está cumpliendo con la meta establecida por lo cual se muestra la existencia de ineficiencia en el mercado financiero de los productos ofertados, lo que permite concluir que el incremento del capital institucional es lento.

- La COAC cancela intereses superiores por préstamos a los fijados en la meta, en este ámbito el costo operativo es alto, es decir no existen adecuadas políticas y procedimientos de cobro en la, determinándose un activo improductivo del 13\% lo que permite determinar que no se está cumpliendo la meta pues este deberá ser menor al $1 \%$.

- Destacándose que las obligaciones externas corresponden al 51\%, por tanto, se requiere mayores fuentes de financiamiento para que la COAC pueda seguir funcionando; en este mismo ámbito el activo total invertido en cartera de créditos es de $70 \%$, al igual que el $4 \%$ del total de los activos son financiados, parámetros que están dentro de las metas esperadas inferiores al 5\%, en ese mismo ámbito se destaca que el aporte de los socios es del 5\%, determinándose que está en el rango meta.

- Existe disponibilidad de capital para cubrir las provisiones y otras pérdidas potenciales, por tanto, el rendimiento de la cartera de crédito está dentro del porcentaje de la meta establecida determinados que el ingreso proveniente de esta cartera lo cual permite cubrir los gastos financieros y operativos en un margen bruto de $15 \%$

- Se determina que la COAC si está estudiando la mejor alternativa de inversión por lo que está obteniendo resultados eficientes, ya que el costo/ rendimiento de los depósitos es del 6\%, siendo mayor a la meta establecida 1,41\%, pagándose un alto interés por las tasas de ahorro 
- Actualmente se está pagado altos intereses por depósitos a plazo lo cual está acompañado del suficiente capital de maniobra para cubrir los gastos y mantener los activos ya que el margen bruto de ingreso fue del 15\%, en este ámbito, los ingresos extraordinarios son mínimos lo que quiere decir que se está cumpliendo con la meta y que los ingresos están proviniendo netamente de las actividades operacionales.

\section{Referencias Bibliográficas}

Amaru, A. (2009). Fundamentos de Administración (Primera ed.). México: Pearson Educación.

Barragán, R. (2014). "El Manual de Competitividad en la gestión de las Organizaciones del Sector Financiero de Economía Popular y Solidario. Caso: Cooperativa de Ahorro y Crédito “Jardín Azuayo”". Tesis de Maestría (Disertación de Administración de Empresas) Recuperada del Repositorio Digital de la Universidad del Azuay.

Barry, R. (2004). Métodos Cuantitativos para los Negocios. México: Pearson Prentice Hall.

Cesar, A. G. (2007). Ratios Financieros. México: Pearson Editorial.

Editorial Océano, (1994). Diccionario de Administración y Finanzas. México.

Fonseca (2019), Evaluación económica y financiera de las Cooperativas de Ahorro y Crédito del Ecuador. Caso Segmento 2 del cantón Ambato provincia de Tungurahua.

Godoy, O. G. (2006). Diccionario de conceptos Económicos y Financieros. México: Pearson Economic.http://www.sri.gob.ec/web/guest/32 recuperado el 22 de agosto de 2017

Gómez, F. (2010). Presente y futuro de las finanzas: situaciones y perspectivas de las finanzas a comienzos del siglo XXI. Revista de contabilidad y dirección, pág. 10, pág.53-78.

Gustavo, T. (2005). Análisis de estados Financieros para la toma de decisiones. LIMA: Pontificia Universidad Católica del Perú "textos universitarios".

James, V. H. (2002). Fundamentos de Administración financiera. México: Pearson Educación.

Martino, M. F. (2004). Diccionario de conceptos Económicos y Financieros. México: Pearson. 
Martínez, A. (2016). Modelo Gestión Financiera, Tesis de Maestría (Disertación de contabilidad y Auditoría) Recuperada del Repositorio Digital de la Universidad Técnica de Ambato

Rodolfo, G., \& Javier, M. (2004). Los métodos de calificación de cartera de crédito y su importancia para los paradigmas de medición de riesgo de crédito. México: LIMUSA, S A.

Salgueiro, Amado. (2011). Indicadores de Gestión y Cuadro de Mando. Madrid: Díaz de Santo.

Santiago, G. E. (2010). TEORIA ECONOMICA DE LA EMPRESA: Fundamentos Teóricos. Madrid: Lavel, S.A.

Santos, D. (2010). La ventaja competitiva. España: Díaz de Santos.

Superintendencia de Bancos y Seguros del Ecuador. (2013). Administración del Riesgo Integral. Libro I Sistema Financiero.

Thomas, C. (2003). Contabilidad. México: Pearson Prentice Hall. 
ISSN: 2600-5859

\section{PARA CITAR EL ARTÍCULO INDEXADO.}

Jácome Tamayo, S. P., Gavilánez Vega, M. I., \& Ibarra Chango, M. del C. (2020). La gestión financiera para el desarrollo competitivo de las cooperativas de ahorro y credito . ConcienciaDigital, 3(1.1), 271-288. https://doi.org/10.33262/concienciadigital.v3i1.1.1154

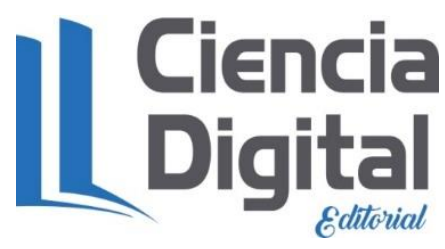

El artículo que se publica es de exclusiva responsabilidad de los autores y no necesariamente reflejan el pensamiento de la Revista Conciencia Digital.

El artículo queda en propiedad de la revista y, por tanto, su publicación parcial y/o total en otro medio tiene que ser autorizado por el director de la Revista Conciencia Digital.
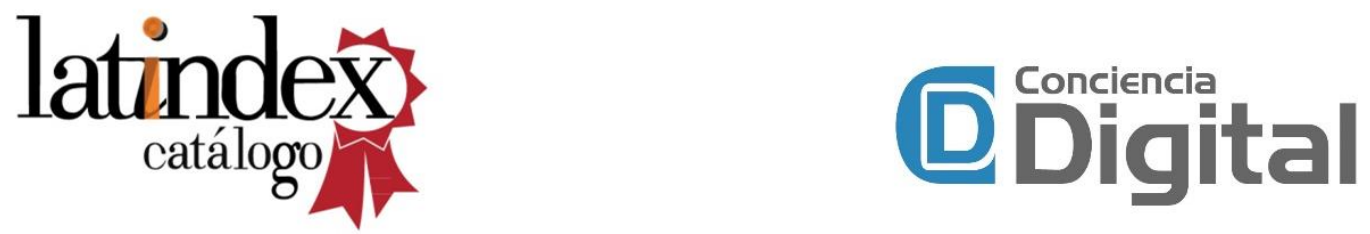